\title{
The effect of a decision aid on informed decision- making in the era of non-invasive prenatal testing: a randomised controlled trial
}

\author{
Lean Beulen ${ }^{\star, 1}$, Michelle van den Berg${ }^{1}$, Brigitte HW Faas², Ilse Feenstra ${ }^{2}$, Michiel Hageman ${ }^{3}$, \\ John MG van Vugt $^{1}$ and Mireille N Bekker ${ }^{1,4}$
}

Early in pregnancy women and their partners face the complex decision on whether or not to participate in prenatal testing for fetal chromosomal abnormalities. Several studies show that the majority of pregnant women currently do not make informed decisions regarding prenatal testing. As the range of prenatal tests is expanding due to the development of new techniques such as non-invasive prenatal testing (NIPT), autonomous reproductive decision-making is increasingly challenging. In this study, a randomised controlled trial was conducted to evaluate the effect of a web-based multimedia decision aid on decision-making regarding prenatal testing. The decision aid provided both written and audiovisual information on prenatal tests currently available, that is, prenatal screening by first-trimester combined testing, NIPT and invasive diagnostic testing through chorionic villus sampling or amniocentesis. Furthermore, it contained values clarification exercises encouraging pregnant women to reflect on the potential harms and benefits of having prenatal tests performed. The use of the decision aid improved informed decisionmaking regarding prenatal testing. Of pregnant women allocated to the intervention group $(n=130) 82.3 \%$ made an informed choice compared with $66.4 \%$ of women in the control group $(n=131), P=0.004$. As the vast majority of pregnant women made decisions consistent with their attitudes towards having prenatal testing performed, this improvement in informed decisionmaking could be attributed mainly to an increase in decision-relevant knowledge. This study shows that the implementation of a web-based multimedia decision aid directly facilitates the ultimate goal of prenatal testing for fetal chromosomal abnormalities, which is enabling informed autonomous reproductive choice.

European Journal of Human Genetics (2016) 24, 1409-1416; doi:10.1038/ejhg.2016.39; published online 18 May 2016

\section{INTRODUCTION}

The main purpose of prenatal testing for fetal chromosomal abnormalities is facilitating autonomous reproductive decision-making for pregnant women and their partners, rather than achieving high uptake rates or prevention of children with chromosomal abnormalities being born. ${ }^{1,2}$ In order to achieve autonomous reproductive decisionmaking, the choice to accept or decline prenatal testing should be informed. An informed choice is made when a woman has adequate decision-relevant knowledge and when her participation or nonparticipation is consistent with her values and attitudes towards undergoing prenatal testing. ${ }^{3,4}$ It requires healthcare professionals to provide accurate, comprehensive and neutral information about the options available. Subsequently, the pregnant woman has to be encouraged to integrate this knowledge with her personal values when reflecting on the possible implications of having prenatal testing performed. Most international evaluations show that the majority of pregnant women currently do not make informed decisions regarding prenatal testing. ${ }^{5,6}$ With the development and introduction of new techniques for screening and diagnosis of fetal chromosomal abnormalities such as microarray analysis and non-invasive prenatal testing (NIPT) using cell-free fetal DNA in maternal plasma, the range of prenatal tests available is ever expanding. Consequently, making an informed decision regarding prenatal testing will be even more challenging.

The use of decision aids has been encouraged to help patients and healthcare professionals share in making informed choices, especially when there are multiple reasonable options, when none of the options has an unequivocal advantage in terms of health outcomes, and when each option has benefits and harms that patients may value differently. Patient decision aids are adjuncts to counselling provided by healthcare professionals. They make the decision explicit, and provide standardised information on available options and their associated outcomes. Furthermore, they help patients clarify their personal values, weigh benefits and harms, and deliberate when making decisions. There is high-quality evidence that decision aids improve the quality of the decisional process as well as the choice made. ${ }^{7}$

This study aims to evaluate the effect of a web-based multimedia decision aid on decision-making regarding prenatal testing for fetal chromosomal abnormalities.

\section{METHODS}

Study design

The study was a randomised controlled trial with allocation to two groups: the intervention group and the control group. Ethical approval was granted by the

\footnotetext{
${ }^{1}$ Department of Obstetrics and Gynaecology, Radboud University Medical Center, Nijmegen, The Netherlands; ${ }^{2}$ Department of Human Genetics, Radboud University Medical Center, Nijmegen, The Netherlands; ${ }^{3}$ PATIENT+ Foundation, The Hague, The Netherlands

*Correspondence: Ms L Beulen, Department of Obstetrics and Gynaecology (791), Radboud University Medical Center, P.0. Box 9101, Nijmegen 6500 HB, The Netherlands. Tel: +31 (0) 24 3619573; Fax: +31 (0) 24 3668597; E-mail: Lean.Beulen@Radboudumc.nl

4Present address: Wilhelmina Children's Hospital Birth Center, Utrecht University Medical Center, Utrecht, The Netherlands.

Received 5 January 2016; revised 14 March 2016; accepted 12 April 2016; published online 18 May 2016
} 
medical ethical committee of the Radboud University Medical Center, Nijmegen, the Netherlands.

\section{Participants}

Participants were recruited from March to October 2015 in 20 prenatal care facilities linked to the Network for Prenatal Diagnosis of the Radboud University Medical Center in Nijmegen, the Netherlands. Pregnant women aged 18 years or older, able to understand the study information and give informed consent were eligible for participation. Women were excluded if they were unable to understand Dutch, or had a multiple pregnancy. Furthermore, to ensure participants' decision-making regarding prenatal testing was not constrained by time pressure, pregnant women $>22$ weeks of gestation were excluded from participation, as in the Netherlands expecting parents can only use prenatal test results for reproductive decision-making when available before 24 weeks of gestation.

\section{Procedures}

Currently in the Netherlands, all pregnant women are offered information on prenatal screening for chromosomal abnormalities in early pregnancy. The information comprises a standardised brochure on prenatal screening by first-trimester combined testing (FCT), published by the National Institute for Public Health and the Environment, and information and counselling provided by a certified obstetric healthcare provider, adhering to national guidelines and quality control. Both brochure and consultation cover the target chromosomal abnormalities trisomy 21 (Down syndrome), trisomy 18 (Edwards syndrome) and trisomy 13 (Patau syndrome), the (age-related) likelihood of having a child with a chromosomal abnormality, prenatal screening by FCT, its results being an estimation of the pregnancy-specific risk, and the follow-up tests available should prenatal screening indicate a high risk for fetal chromosomal abnormalities. Furthermore, the possible implications of prenatal testing are discussed, and expectant parents are encouraged to make personal reproductive choices.

Following the first counselling session on prenatal screening for chromosomal abnormalities, eligible pregnant women were asked to participate in the study. After obtaining informed consent, participants were allocated to the control or intervention group by a computer-generated randomisation. Pregnant women randomised to the control group received standard prenatal care. Pregnant women randomised to the intervention group were additionally granted access to a web-based multimedia decision aid on prenatal testing.

The web-based multimedia decision aid was developed according to the International Patient Decision Aid Standards (IPDAS). ${ }^{8,9}$ It provides information on chromosomal abnormalities, prenatal screening by FCT, and the prenatal tests offered in case of a high risk for fetal chromosomal abnormalities, that is, NIPT or invasive diagnostic testing through chorionic villus sampling or amniocentesis. Infographics, 2D and 3D animations were specifically designed for this decision aid to explain and visualise the prenatal testing trajectory, the concept of risk, the difference between a low-risk and high-risk result, and the key characteristics and test procedures of prenatal tests available. Subsequently, pregnant women were asked to test their understanding of the information provided, by means of multiple choice questions on key issues presented in the decision aid, with immediate confirmation and correction of correct and incorrect responses, respectively. Furthermore, in a values clarification exercise women were encouraged to reflect on the value of information provided by prenatal testing and to weigh the potential benefits and disadvantages of having prenatal tests performed. ${ }^{10}$

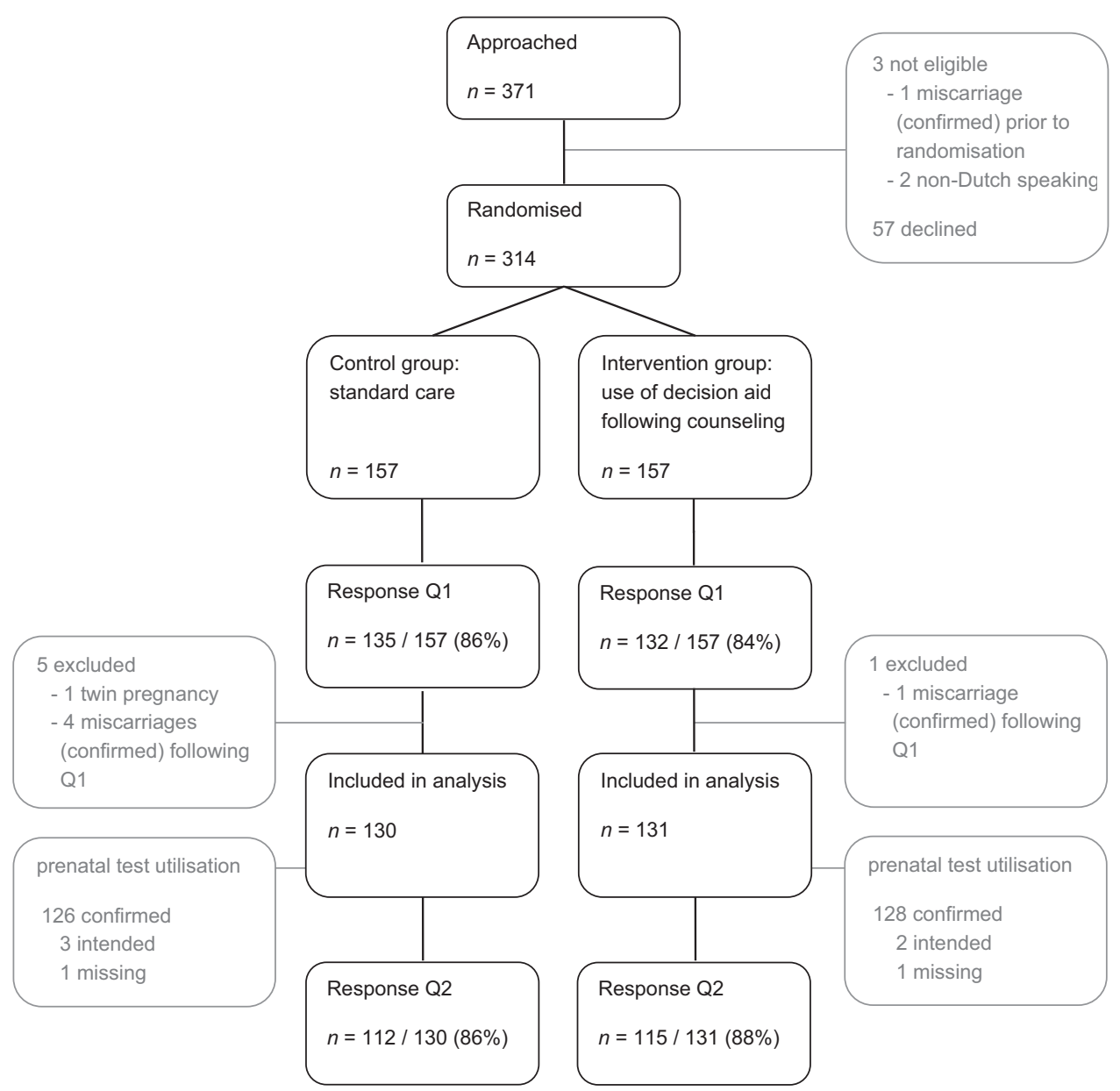

Figure 1 Study participants and flow. Q1, questionnaire 1; Q2, questionnaire 2. 
Pregnant women randomised to the intervention group were sent a personal link providing access to the web-based multimedia decision aid for the duration of 1 week. One week after randomisation, during the process of decisionmaking, participants were invited to complete the first of two web-based questionnaires (Q1). Thus, participants randomised to the intervention group did not have the decision aid available while completing this questionnaire. The invitation to complete a follow-up questionnaire was sent at around 28 weeks of gestation, when the decisional process was completed and, where applicable, prenatal testing for chromosomal abnormalities was performed and the outcome was known $(\mathrm{Q} 2)$.

\section{Outcomes and measures}

The primary outcome is informed decision-making regarding prenatal testing. Secondary outcomes include knowledge, attitudes, prenatal test utilisation, value-consistency, decision conflict, decisional regret and anxiety.

Informed decision-making was assessed using a scale based on the Multidimensional Measure of Informed Choice, which combines knowledge with value-consistency comprising attitudes and prenatal test utilisation into a single measure. ${ }^{3,4}$ The original scale was modified based on a systematic review of international literature, individual semi-structured interviews conducted with pregnant women and healthcare professionals, and discussion by a panel of experts in the field of perinatology and (clinical) genetics.

Knowledge about prenatal testing for fetal chromosomal abnormalities was measured using a knowledge scale that contained 19 statements, with the response options of 'true', 'false' or 'do not know' (Supplementary Table 1). The scale was based on a knowledge measure developed and validated to evaluate the effectiveness of the provision of information and counselling in reaching sufficient knowledge regarding prenatal screening using FCT. ${ }^{11,12}$ The content of the original measure was based on a generic list of domains considered to be essential for an informed choice, including the condition being screened for and characteristics of the screening programme. To cover all prenatal screening and diagnostic tests currently available in the programme, additional items were developed specifically for this trial, based on the standardised national information material available. Total knowledge scores were obtained by summing up the number of correct responses. Scores of $\geqslant 12$, representing $>60 \%$ correct responses, were determined to indicate sufficient knowledge. ${ }^{12}$ In this sample, the modified knowledge scale was internally consistent with a Cronbach's alpha of 0.69 .

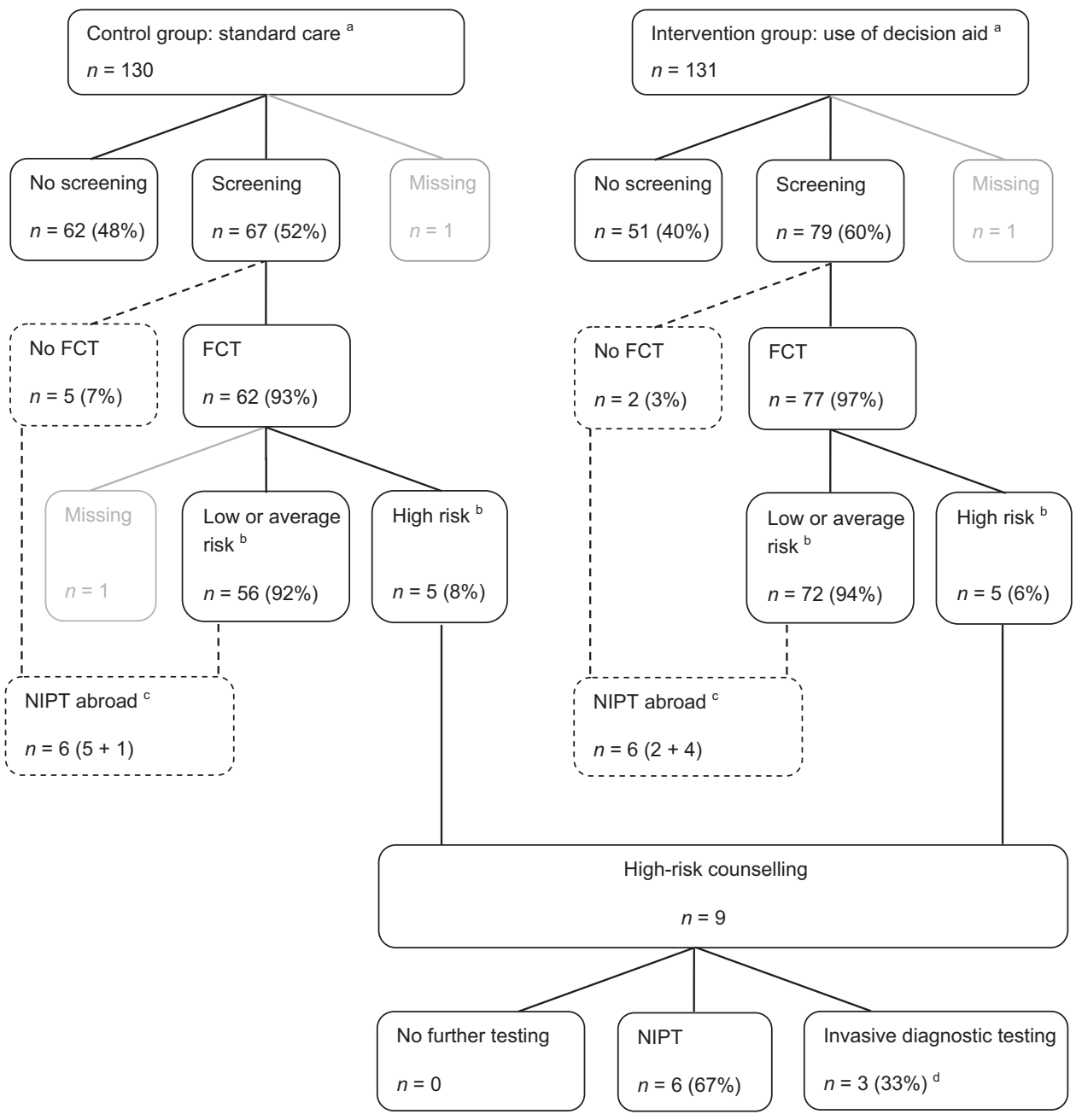

Figure 2 Prenatal test utilisation of study participants. Dashed lines indicate pathways outside the current programme for prenatal screening and diagnosis

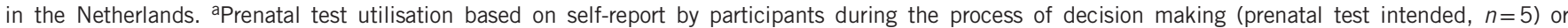
when the decisional process was completed and, where applicable, prenatal testing for chromosomal abnormalities was performed and the outcome was known (prenatal test confirmed, $n=254$ ). bCut-off risk FCT 1: $\leq 200$. ' Offering NIPT as primary screening test is currently prohibited in The Netherlands, but available in neighbouring countries. dTwo participants had invasive diagnostic testing performed after FCT had revealed a nuchal translucency $\geq 3.5$ mm. One participant had invasive diagnostic testing performed after FCT had shown maternal serum markers (PAPP-A and free $\beta$ hCG) to be suggestive for fetal triploidy. FCT, first-trimester combined test; NIPT, non-invasive prenatal test. 
Attitudes were measured using an attitude scale that contained five bipolar adjective pairs (Supplementary Table 2). The selected adjective pairs were all validated in a prenatal setting and considered relevant for decision-making regarding both prenatal screening and diagnostic testing., ${ }^{3,4,12-14}$ Participants were directed to consider responses in terms of their attitudes towards having prenatal screening by FCT performed, towards having invasive diagnostic testing performed, and towards having NIPT performed (alpha 0.84, 0.81 and 0.88 , respectively). The midpoint of the scale equals a neutral attitude. Attitudes scores above and below this midpoint indicated positive and negative attitudes, respectively.

Intended and actually performed prenatal testing was self-reported by participants in Q1 (intended) and Q2 (confirmed).

Attitudes were combined with prenatal test utilisation to assess whether decision-making regarding prenatal testing was value-consistent. Currently in the Netherlands, prenatal screening by FCT is the only prenatal test available to the vast majority of pregnant women, that is, those without a personal or family

Table 1 Characteristics of participating pregnant women

\begin{tabular}{ccccc}
$\begin{array}{c}\text { Control } \\
(n=130)\end{array}$ & \multicolumn{2}{c}{$\begin{array}{c}\text { Decision aid } \\
(n=131)\end{array}$} \\
$n$ & $\%$ & $n$ & $\%$ & $P$ \\
\hline
\end{tabular}

Age

Mean (SD), range

31.0 (3.8), 23-41 31.3 (4.0), 20-44 0.524

Highest educational qualification ${ }^{a}$

$\begin{array}{lrrrc}\text { Low } & 9 & 6.9 & 7 & 5.4 \\ \text { Intermediate } & 39 & 30.0 & 33 & 25.4 \\ \text { High } & 82 & 63.1 & 90 & 69.2\end{array}$

Missing

1

Net monthly household income ${ }^{b}$

$$
\begin{aligned}
& \leqslant € 2999 \\
& \geqslant € 3000 \\
& \text { Missing }
\end{aligned}
$$

36

$$
28.3
$$

35

27.6

71.7

92

72.4

Religiosity

Religious
Not religious

53

40.8

4

0.571

77

59.2

54

41.2

0.941

Background

Dutch

Non-Dutch

Gestational age (at Q1)

Mean (SD), range

$12.5(2.4), 8-19 \quad 12.0(2.1), 8-22 \quad 0.083$

First pregnancy

$\begin{array}{lrrrrr}\text { Yes } & 54 & 41.5 & 61 & 46.6 & 0.413 \\ \text { No } & 76 & 58.5 & 70 & 53.4 & \\ & & & & & \\ \text { Parity } & 71 & 54.6 & 77 & 58.8 & 0.497 \\ \quad \text { Nulliparous } & 59 & 45.4 & 54 & 41.2 & \\ \begin{array}{l}\text { Parous } \\ \text { Prenatal test in (any) previous pregnancy }\end{array} & & & & \\ \text { Yes } & 24 & 31.6 & 17 & 24.3 & 0.327 \\ \text { No } & 52 & 68.4 & 53 & 75.7 & \end{array}$

aLow: primary school, lower general secondary education, lower vocational education; intermediate: higher general secondary education, pre-university secondary education, higher vocational education; high: higher professional education, university.

butch average net monthly household income in 2014: € 2850 (Statistics Netherlands,

http://statline.cbs.nl, retrieved January 2016).

cReported for those women with a previous pregnancy $(n=146)$. history of chromosomal abnormalities. Hence, when negative attitudes towards prenatal screening by FCT involved having no prenatal screening performed, or positive attitudes towards prenatal screening by FCT were acted upon by accepting prenatal screening, the participant was classified as having made a value-consistent decision. Although offering NIPT as primary screening test is currently prohibited in the Netherlands, it is available in neighbouring countries. Therefore, women who had NIPT as primary screening test performed were also classified as having made a value-consistent decision, provided that their attitude towards NIPT was positive.

Participants were classified as having made an informed choice if their decision to accept or decline prenatal screening was based on sufficient knowledge, and reflected their values.

Levels of decisional conflict were measured during the process of decisionmaking, using the Decisional Conflict Scale (DCS). ${ }^{15,16}$ The DCS evaluates participants' perspectives of how certain they feel about the decision and their perceived autonomy in decision-making. It is divided into five subscales: feeling informed, values clarity, feeling supported, uncertainty and perceived effectiveness of decision-making (alpha 0.95). Decision regret was measured after the completion of the process of decision-making, using the Decision Regret Scale (DRS). ${ }^{17}$ It evaluates distress or remorse after making a decision (alpha 0.83).

Levels of anxiety were measured using the six-item short form of the state scale of the Spielberger State Trait Anxiety Inventory (STAI) (alpha 0.85 and 0.76$){ }^{18,19}$

\section{Sample size}

To detect a difference of $15 \%$ in informed choice (70\% in control group versus $85 \%$ in intervention group) with $80 \%$ power at a 5\% level of significance, 121 pregnant women were required in each group. This difference was based on studies assessing informed choice regarding prenatal screening in the Netherlands and is considered to be clinically relevant. ${ }^{12-14}$

\section{Statistical analysis}

Group differences were assessed using $\chi^{2}$ tests for categorical variables and independent-samples $t$-tests for continuous variables. Conditional logistic regression analysis was conducted to determine which independent variables were significant predictors of informed decision-making and participation in prenatal testing. Analyses were performed using SPSS (version 22.0 for Windows, SPSS Inc., Chicago, IL, USA).

\section{RESULTS}

In total, 314 pregnant women consented to participate in the trial, of whom 157 were randomly allocated to the intervention group and 157 to the control group. The questionnaire was completed by 267 participants (response $85.0 \%$ ). In total, six pregnant women were excluded from analysis because a non-viable or multiple pregnancy was diagnosed after randomisation. The 261 remaining women (130 randomised to the control group and 131 randomised to the intervention group) were

\begin{tabular}{|c|c|c|c|c|c|}
\hline & \multicolumn{2}{|c|}{$\begin{array}{l}\text { Control } \\
(n=130)\end{array}$} & \multicolumn{2}{|c|}{$\begin{array}{c}\text { aid } \\
(n=131)\end{array}$} & \multirow[b]{2}{*}{$\mathrm{P}$} \\
\hline & $\mathrm{n}$ & $\%$ & $\mathrm{n}$ & $\%$ & \\
\hline \multicolumn{6}{|l|}{ Informed choice } \\
\hline Knowledge sufficient, choice value-consistent & 83 & 66.4 & 102 & 82.3 & 0.004 \\
\hline \multicolumn{6}{|l|}{ Choice not informed } \\
\hline Knowledge insufficient & 35 & 28.0 & 13 & 10.5 & \\
\hline Knowledge insufficient, choice value-inconsistent & 3 & 2.4 & 2 & 1.6 & \\
\hline Knowledge sufficient, choice value-inconsistent & 4 & 3.2 & 7 & 5.6 & \\
\hline Missing & 5 & & 7 & & \\
\hline
\end{tabular}
included in analysis (Figure 1). There were no statistically significant differences in the socio-demographic and obstetric characteristics of both

Table 2 Primary outcome-informed decision-making 
groups (Table 1). Of the 259 pregnant women with known prenatal test utilisation, $146(56.4 \%)$ decided to have prenatal screening performed: 91.8\% $(n=134)$ decided to accept FCT, $4.8 \%(n=7)$ chose to have NIPT performed abroad, and 3.4\% $(n=5)$ chose to accept FCT, as well as have NIPT performed abroad (Figure 2).

The use of a web-based multimedia decision aid in addition to usual care improved informed decision-making regarding prenatal testing (Table 2). In the intervention group, $82.3 \%$ of participating pregnant women made an informed choice, compared with $66.4 \%$ in the control group $(P=0.004)$.

When a choice was not informed this was predominantly due to insufficient knowledge (Tables 2 and 3). Women allocated to the intervention group scored significantly higher on the knowledge scale (14.9 versus $12.8, P<0.001$ ). This resulted in more women being classified as having sufficient knowledge in the intervention group than in the control group ( 88.5 versus $70.8 \%, P<0.001)$. The decision aid was especially effective in increasing knowledge of prenatal tests available for those pregnancies complicated by a high risk for fetal chromosomal abnormalities (Supplementary Table 1).

There were neither statistically significant differences in proportions of women having positive or negative attitudes towards having prenatal tests performed (Table 3), nor in actual prenatal test utilisation (Figure 2). The vast majority of women made a value-consistent decision (92.7\% versus $94.2 \%, P=0.641)$. Of the 16 participants whose

Table 3 Secondary outcomes—-knowledge, attitudes, value-consistency, decisional conflict, decision regret and anxiety

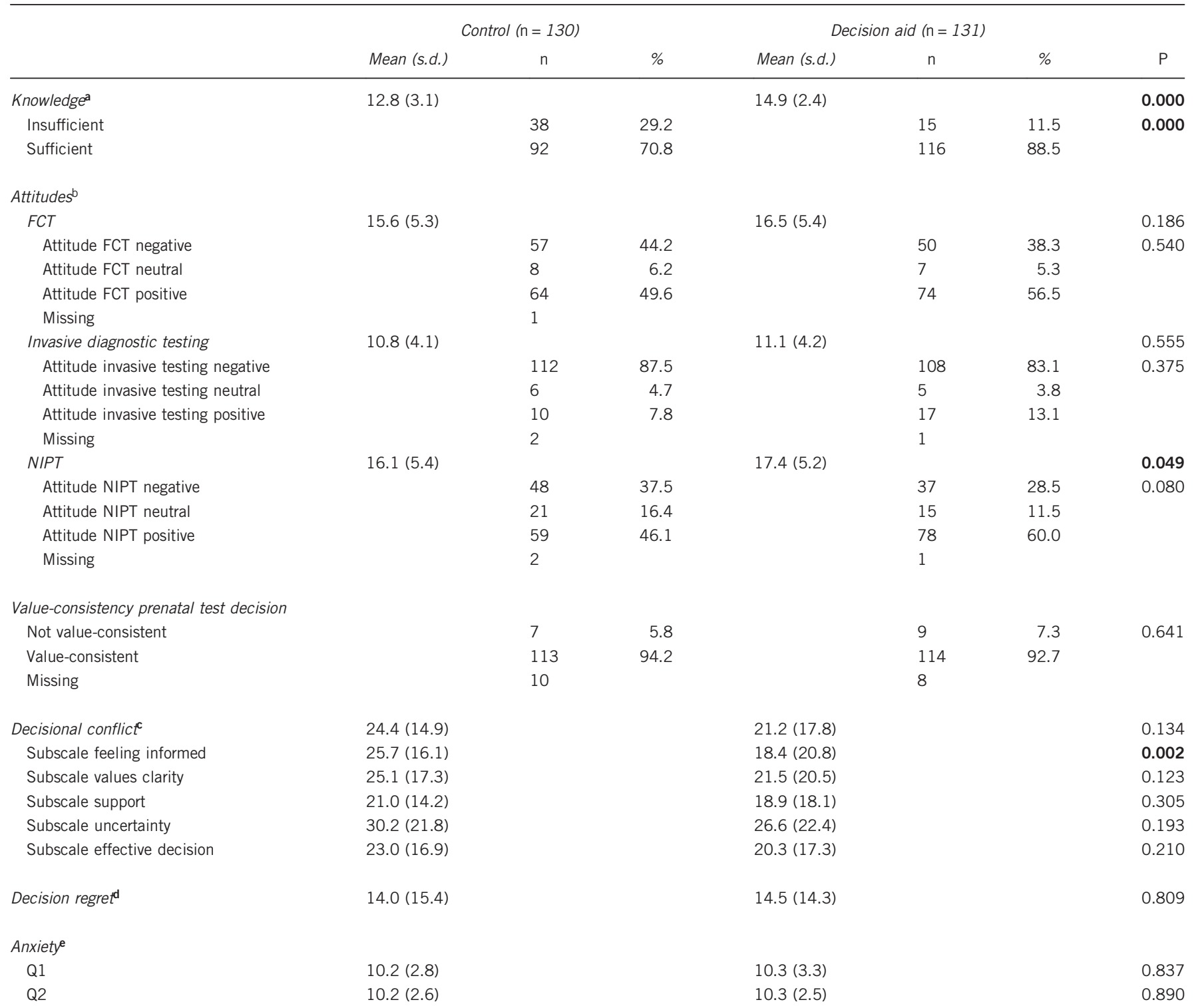

aThe knowledge scale contains 19 statements. Knowledge scores were obtained by summing up the number of correct responses, giving a minimum score of 0 and a maximum score of 19 . Participants with a score of $\geq 12$, representing $>60 \%$ correct responses, were classified as having sufficient knowledge.

bThe attitude scale contains five bipolar adjective pairs, each scored on a 5-point Likert scale. Attitude scores were obtained by summing up scores, giving a minimum score of 5 and a maximum score of 25. Attitudes scores $>15$ and $<15$ indicated positive and negative attitudes, respectively.

${ }^{c}$ The Decisional Conflict Scale (DCS) contains 16 items scored on a 5-point Likert scale. All subscales as well as the overall DCS range from 0 to 100 , with higher scores indicating greater decisional conflict. Scores $<25.0$ are associated with implementing decisions and scores $>37.5$ are associated with feeling unsure about implementation of the decision.

dThe Decision Regret Scale (DRS) contains five items scored on a 5-point Likert scale. It ranges from 0 to 100 , with higher scores indicating greater decision regret.

${ }^{\mathrm{e}}$ The short form of the state scale of the Spielberger State Trait Anxiety Inventory (STAI) contains six items. It ranges from 6 to 24 , with higher scores indicating greater levels of anxiety.

The bold is used to indicate statistically significant differences $(P$-values $<0.05)$ between control and intervention groups. 
decision-making was value-inconsistent, $50.0 \%$ declined prenatal screening despite having positive attitudes and $50.0 \%$ accepted prenatal screening despite having negative attitudes towards having prenatal screening performed (Tables 2 and 3). Women allocated to the intervention group had lower decisional conflict related to feeling uninformed (18.4 versus 25.7, $P=0.002$ ). No statistically significant differences were found in total decisional conflict (21.2 versus $24.4, P=0.134)$, decision regret (14.5 versus 14.0, $P=0.809$ ) or anxiety (Q1 10.3 versus $10.2, P=0.837$, Q2 10.3 versus 10.2, $P=0.890)$.

Logistic regression analysis revealed that advanced maternal age (OR 1.09 per year, 95\% CI 1.00-1.18, $P=0.046$ ), a high level of education (OR 3.53, 95\% CI 1.90-6.57, $P<0.001$ ), and allocation to the intervention group, that is, having access to the web-based multimedia decision aid (OR 2.39, 95\% CI 1.27-4.49, $P=0.007$ ), were the most important independent predictors of informed decisionmaking (Table 4). Although univariate analysis indicated maternal age, level of education, net monthly household income and religiosity to be significantly associated with prenatal test utilisation, multivariate logistic regression analysis revealed that the combination of advanced maternal age (OR 1.13 per year, 95\% CI 1.06-1.22, $P=0.001$ ), a high level of education (OR 2.37, 95\% CI 1.36-4.12, $P=0.002$ ) and a non-Dutch background (OR 2.63, 95\% CI 0.95-7.25, $P=0.062$ ) best predicted participation in prenatal testing (Table 4 and Supplementary Table 3). Women who had prenatal tests performed had higher knowledge scores (14.4 versus $13.2, P=0.001)$ and thus more often made an informed choice $(80.3 \%$ versus $66.4 \%, P=0.013)$ than those who did not (Supplementary Tables 4 and 5).

\section{DISCUSSION}

Early in pregnancy women and their partners face the complex decision on whether or not to participate in prenatal testing for fetal chromosomal abnormalities. The majority of pregnant women currently do not make informed decisions regarding prenatal testing. ${ }^{5,6}$ This study shows that the use of a web-based multimedia decision aid significantly increases pregnant women's informed autonomous reproductive decision-making.

The web-based multimedia decision aid evaluated in the present work is the first decision aid to include information on NIPT as one of the prenatal screening and diagnostic tests currently available. Moreover, it is the first evaluation of informed decision-making regarding prenatal testing in the general pregnant population since NIPT has been introduced in prenatal care. Previous research had already shown that the vast majority of pregnant women at intermediate or high risk for fetal aneuploidy following prenatal screening and counselled on having NIPT performed as a contingent screening test make informed decisions. ${ }^{20}$ In the present study, decision-making regarding prenatal testing was evaluated at the start of the testing trajectory, and was also found to be informed. An interesting finding of this trial was the large proportion of pregnant women having negative attitudes towards

Table 4 Logistic regression analysis estimating the probability of informed decision-making and participation in prenatal testing

\begin{tabular}{|c|c|c|c|c|c|c|c|c|}
\hline \multirow[b]{3}{*}{ Variable } & \multicolumn{4}{|c|}{ Informed decision-making } & \multicolumn{4}{|c|}{ Prenatal test utilisation } \\
\hline & \multicolumn{2}{|c|}{ Univariate analysis } & \multicolumn{2}{|c|}{ Multivariate analysis } & \multicolumn{2}{|c|}{ Univariate analysis } & \multicolumn{2}{|c|}{ Multivariate analysis } \\
\hline & OR $(95 \% \mathrm{Cl})$ & $P$ & OR $(95 \% \mathrm{Cl})$ & $\mathrm{P}$ & OR $(95 \%$ Cl) & $\mathrm{P}$ & OR $(95 \% \mathrm{Cl})$ & $\mathrm{P}$ \\
\hline \multicolumn{9}{|l|}{ Age } \\
\hline Increase of 1 year & $1.11(1.02,1.20)$ & 0.013 & $1.09(1.00,1.18)$ & 0.046 & $1.15(1.07,1.23)$ & $<0.001$ & $1.13(1.06,1.22)$ & 0.001 \\
\hline \multicolumn{9}{|c|}{ Highest educational qualification ${ }^{\mathrm{a}}$} \\
\hline Low or intermediate & 1 & & 1 & & 1 & & 1 & \\
\hline High & $4.02(2.22,7.31)$ & $<0.001$ & $3.53(1.90,6.57)$ & $<0.001$ & $2.61(1.54,4.42)$ & $<0.001$ & $2.37(1.36,4.12)$ & 0.002 \\
\hline \multicolumn{9}{|c|}{ Net monthly household income ${ }^{\mathrm{b}}$} \\
\hline$\leq € 2999$ & 1 & & & & 1 & & & \\
\hline$\geq € 3000$ & $1.83(0.99,3.39)$ & 0.055 & & & $2.35(1.34,4.12)$ & 0.003 & & \\
\hline \multicolumn{9}{|l|}{ Religiosity } \\
\hline Not religious & 1 & & & & 1 & & & \\
\hline Religious & $0.59(0.33,1.04)$ & 0.067 & & & $0.60(0.37,1.00)$ & 0.049 & & \\
\hline \multicolumn{9}{|l|}{ Background } \\
\hline Dutch & 1 & & & & 1 & & 1 & \\
\hline Non-Dutch & $0.66(0.67,1.63)$ & 0.371 & & & $2.51(0.96,6.54)$ & 0.060 & $2.63(0.95,7.25)$ & 0.062 \\
\hline \multicolumn{9}{|l|}{ Parity } \\
\hline Nulliparous & 1 & & & & 1 & & & \\
\hline Parous & $0.92(0.52,1.63)$ & 0.920 & & & $0.70(0.42,1.14)$ & 0.151 & & \\
\hline \multicolumn{9}{|l|}{ Decision aid } \\
\hline Control & 1 & & 1 & & 1 & & & \\
\hline Intervention & $2.35(1.30,4.24)$ & 0.005 & $2.39(1.27,4.49)$ & 0.007 & $1.43(0.88,2.35)$ & 0.152 & & \\
\hline
\end{tabular}

aLow: primary school, lower general secondary education, lower vocational education. Intermediate: higher general secondary education, pre-university secondary education, higher vocational education. High: higher professional education, university.

bDutch average net monthly household income in 2014: € 2850 (Statistics Netherlands, http://statline.cbs.nl, retrieved January 2016).

The bold is used to indicate statistically significant differences ( $P$-values $<0.05)$ between control and intervention groups. 
having NIPT performed, that is, $32 \%$ of all the participants. Both findings are in line with previous studies conducted in the Netherlands, where many women make an informed choice not to participate in prenatal screening, and some would decline prenatal testing for chromosomal abnormalities even when offered a (near-) perfect prenatal test. ${ }^{12-14,21}$

Other studies evaluating the effect of patient decision aids on decisionmaking regarding prenatal testing for fetal chromosomal abnormalities have been published. Previous studies have reported on the application of booklets with worksheets, ${ }^{22,23}$ information films, ${ }^{24,25}$ and interactive computer programmes. ${ }^{26-28}$ Although results from these studies cannot easily be compared because of heterogeneity in settings, interventions and outcome measures, most have general findings that correspond with the results of the present study. Nearly all found the application of a decision aid to increase pregnant women's decision-relevant knowledge ${ }^{22-27}$ and informed decision-making. ${ }^{23,25}$ In this trial, high baseline levels of informed decision-making in the control group notwithstanding the application of the web-based multimedia decision aid resulted in an increase in informed decision-making that was slightly higher than the effect found in previous evaluations. ${ }^{23,25}$

As the information provided in a decision aid is standardised, thus not affected by the counsellor's knowledge and attitudes, it is more likely to be accurate and non-directive than traditional methods of counselling. A web-based multimedia decision aid can be organised in several levels of information, for example, from basic to advanced, or from prenatal screening to diagnostic testing, reflecting the different steps of the testing trajectory. This allows pregnant women and their partners to control the amount and content of the information provided and enables processing of information at their own pace, at a time and place they find convenient. This is especially important as couples face increasingly complex decisions as a result of the ever expanding range of prenatal tests available. ${ }^{29}$ Adequately informing pregnant women on the test characteristics of these tests, that is, the conditions tested for, and their diagnostic accuracy, limitations and potential harms, is a prerequisite for informed decisionmaking. This, however, entails a threat of so-called information overload and decisional conflict, which could be alleviated by the use of a web-based multimedia decision aid.?

Ideally, when implementing the decision aid in prenatal care, the couple should be able and encouraged to access the decision aid before the consultation with their obstetric healthcare provider. Given its proven effectiveness in increasing decision-relevant knowledge, it is likely that such preparation will result in less time spent on the provision of routine information. This provides an opportunity for a shift towards patient-centred counselling and individualised decision-making support, that is, the identification of remaining knowledge gaps, and the exploration of pregnant women's personal values and attitudes towards prenatal testing and its possible implications. As a result, a so-called 'routinisation' of prenatal testing will be less likely to occur.

Some limitations of this study can be recognised. The pregnant women participating in this study were predominantly of Dutch background and had high levels of education and family income. Furthermore, this trial showed above-average rates of participation in prenatal testing in both control and intervention group, which may indicate that women interested in prenatal testing may have been more willing to participate in the trial. As a result, the generalizability of the findings may be limited.

\section{CONCLUSION}

This study shows that the use of a web-based multimedia decision aid in addition to usual prenatal care improves informed decision-making regarding participation in prenatal testing for fetal chromosomal abnormalities. Findings suggest that the implementation of such a decision aid would therefore directly facilitate the ultimate goal of the national programme for prenatal screening and diagnosis, which is enabling informed, autonomous reproductive choice.

\section{CONFLICT OF INTEREST}

The authors declare no conflict of interest.

\section{ACKNOWLEDGEMENTS}

We are grateful to all pregnant women and healthcare professionals who have participated in this study. This study was financially supported by the Foundation for Prenatal Screening in the Nijmegen Region.

1 Health Council of the Netherlands. Prenatal screening: Down syndrome, neural tube defects, routine ultrasonography. The Hague: Health Council of the Netherlands, 2001; publication no. 2001/11.

2 De Jong A, Dondorp WJ, Frints SGM, De Die-Smulders CEM, De Wert GMWR: Advances in prenatal screening: the ethical dimension. Nat Rev Genet 2011; 12: 657-663.

3 Marteau TM, Dormandy E, Michie S: A measure of informed choice. Health Expect 2001; 4: 99-108.

4 Michie A, Dormandy E, Marteau TM: The multi-dimensional measure of informed choice: a validation study. Patient Educ Couns 2002; 48: 87-91.

5 Green JM, Hewison J, Bekker HL, Bryant LD, Cuckle HS: Psychosocial aspects of genetic screening of pregnant women and newborns: a systematic review. Health Technol Assess 2004; 8: 1-128.

6 Ames AG, Metcalfe SA, Archibald AD, Duncan RE, Emery J: Measuring informed choice in population-based reproductive genetic screening: a systematic review. Eur J Human Genet 2015; 23: 8-21.

7 Stacey D, Légare F, Col NF et al: Decision aids for people facing health treatment or screening decisions. Cochrane Database Syst Rev 2014, issue 1.

8 Elwyn G, O'Connor AM, Stacey D et al: Developing a quality criteria framework for patient decision aids: online international Delphi consensus process. BMJ 2006; 333: 417

9 Elwyn G, O'Connor AM, Bennett C et al: Assessing the quality of decision support technologies using the international patient decision aid standards instrument (IPDASi). PLoS One 2009; 4: e4705.

10 Web-based multimedia decision aid on prenatal testing. Available at http://www. keuzehulp.info/radboud/pnt (accessed on 03 March 2016).

11 Schoonen HM, van Agt HM, Essink-Bot ML, Wildschut HI, Steegers EA, de Koning HJ: Informed decision-making in prenatal screening for Down's syndrome: what knowledge is relevant? Patient Educ Couns 2011; 84: 265-270.

12 Schoonen M, Wildschut H, Essink-Bot ML, Peters I, Steegers E, de Koning H: The provision of information and informed decision-making on prenatal screening for Down syndrome: a questionnaire-based and register-based survey in a non-selected population. Patient Educ Couns 2012; 87: 351-359.

13 van den Berg M, Timmermans DRM, Ten Kate LP, van Vugt JMG, van der Wal G: Are pregnant women making informed choices about prenatal screening? Genet Med 2005; 7: 332-338.

14 Fransen MP, Essink-Bot ML, Vogel I, Mackenbach JP, Steegers EA, Wildschut HI: Ethnic differences in informed decision-making about prenatal screening for Down's syndrome. J Epidemiol Community Health 2010; 64: 262-268.

15 O'Connor AM: Validation of a decisional conflict scale. Med Decis Making 1995; 15: 25-30.

16 Koedoot N, Molenaar S, Oosterveld P et al: The decisional conflict scale: further validation in two samples of Dutch oncology patients. Patient Educ Couns 2001; 45 . 187-193.

17 Brehaut JC, O'Connor AM, Wood TJ et al: Validation of a decision regret scale. Med Decis Making 2003; 23: 281-292.

18 Marteau TM, Bekker H: The development of a six-item short-form of the state scale of the Spielberger state-trait anxiety inventory (STAI). Br J Clin Psychol 1992; 31: 301-306.

19 Van der Bij AK, de Weerd S, Cikot RJ, Steegers EA, Braspenning JC: Validation of the Dutch short form of the state scale of the Spielberger State-Trait Anxiety inventory: considerations for usage in screening outcomes. Community Genet 2003; 6: 84-87.

20 Lewis C, Hill M, Skirton H, Chitty LS: Development and validation of a measure of informed choice for women undergoing non-invasive prenatal testing for aneuploidy. Eur J Hum Genet 2016; 24: 809-816.

21 Beulen L, Grutters JP, Faas BH et al: Women's and healthcare professionals' preferences for prenatal testing: a discrete choice experiment. Prenat Diagn 2015; 35: 549-557. 
22 Hunter AGW, Cappelli M, Humphreys L et al: A randomized trial comparing alternative approaches to prenatal diagnosis counseling in advanced maternal age patients. Clin Genet 2005; 67: 303-313.

23 Nagle C, Gunn J, Bell R et al: Use of a decision aid for prenatal testing of fetal abnormalities to improve women's informed decision making: a cluster randomised controlled trial. BJOG 2008; 115: 339-347.

24 Hewison J, Cuckle $\mathrm{H}$, Baillie $\mathrm{C}$ et al: Use of videotapes for viewing at home to inform choice in Down syndrome screening: a randomised controlled trial. Prenat Diagn 2001; 21: $146-149$.

25 Björklund U, Marsk A, Levin C, Öhman SG: Audiovisual information affects informed choice and experience of information in antenatal Down syndrome screening: a randomized controlled trial. Patient Educ Couns 2012; 86: 390-395.
26 Kuppermann M, Norton ME, Gates $\mathrm{E}$ et al: Computerized prenatal genetic testing decision-assisting tool: a randomized controlled trial. Obstet Gynecol 2009; 113 : 53-63.

27 Kuppermann M, Pena S, Bishop JT et al: Effect of enhanced information, values clarification, and removal of financial barriers on use of prenatal genetic testing: a randomized clinical trial. JAMA 2014; 312: 1210-1217.

28 Skjoth MM, Draburg E, Lamont RF et al: Informed choice about Down syndrome screening - effect of an eHealth tool: a randomized controlled trial. Acta Obstet Gynecol Scand 2015; 94: 1327-1336.

29 Faas $\mathrm{BH}$ : Prenatal genetic care: debates and considerations of the past, present and future. Expert Opin Biol Ther 2015; 15: 1101-1105.

Supplementary Information accompanies this paper on European Journal of Human Genetics website (http://www.nature.com/ejhg) 